\title{
Epidemiological, clinical, and genotype characterization of spinocerebellar ataxia type in families in Buriram province, northeast Thailand
}

\author{
Suppachok Wetchaphanphesat ${ }^{1, *}$, Anek Mungaomklang ${ }^{2}$, Chutima Papsing ${ }^{3}$, Teeratorn Pulkes ${ }^{3}$
}

\begin{abstract}
Background: In Thais, the most prevalent type of spinocerebellar ataxia (SCA) is type 3, most commonly known as Machado-Joseph disease (MJD), followed by SCA type 1 (SCA1), SCA2, and SCA6.

Objectives: To describe the epidemiological, clinical, and genotypic features of SCA in northeastern Thailand and to study 2 associations: between syndromic features and the genotype of SCA, and between health determinants and scores on the scale for the assessment and rating of ataxia (SARA).

Methods: We conducted a cross-sectional study of 24 patients with autosomal dominant SCA from 13 families recruited from Buriram province in northeast Thailand between December 2009 and January 2014. Patients provided a clinical history and were examined by a neurologist. DNA was extracted from the peripheral blood of each patient. We analyzed associations between the type of SCA and sex, age, family history, clinical features, any underlying disease, age at onset, body weight, smoking status, family history, alcohol consumption, head injury history, and SARA.

Results: Seven of the families were positive for SCA1 and 6 for MJD. There were 24 index patients from these autosomal dominant SCA families, including 13 with SCA1 and 11 with MJD. Their average age was 43.7 years (range 20-72 years), whereas their average age at disease onset was 36.9 years (range 18-59 years). Pyramidal signs between MJD and SCA1 were not significantly different. Extrapyramidal features appeared uncommon. Horizontal nystagmus and upward gaze paresis were significantly associated with MJD. There were no significant differences in demographic data between the groups with SARA scores $\geq 15$ or $<15$.

Conclusions: MJD and SCA1 were the 2 adult-onset cerebellar degenerative diseases found in Buriram province. Clinical clues for differentiating between them were upward gaze paresis and horizontal nystagmus, which were significantly more common in MJD.
\end{abstract}

Keywords: clinical characteristic, epidemiology, northeast Thailand, spinocerebellar ataxia type

Spinocerebellar ataxias (SCAs) are a group of neurodegenerative, motor-coordination diseases caused by autosomal dominant disorders of the cerebellum and its afferent or efferent pathways. Among them, spinocerebellar ataxia type 1 (SCA1), spinocerebellar ataxia type 2 (SCA2), spinocerebellar ataxia type 3 (SCA3), spinocerebellar ataxia

*Correspondence to: Suppachok Wetchaphanphesat, Department of Medicine, Division of Neurology, Buriram Hospital, Buriram 31000,

Thailand, e-mail: suppachok@health.moph.go.th

'Department of Medicine, Buriram Hospital, Buriram 31000, Thailand

2Department of Occupational Health, Debaratana Nakhon Ratchasima Hospital, Nakhon Ratchasima 30280, Thailand

${ }^{3}$ Department of Medicine, Faculty of Medicine, Ramathibodi Hospital, Mahidol University, Bangkok 10400, Thailand

כ Open Access. ๑ 2017 Suppachok Wetchaphanphesat et al., published by Sciendo. (๔) BY-Nc-ND This work is licensed

under the Creative Commons Attribution NonCommercial-NoDerivatives 4.0 License. 
type 6 (SCA6), spinocerebellar ataxia type 7 (SCA7), and spinocerebellar ataxia type 17 (SCA17), as well as dentatorubral-pallidoluysian atrophy (DRPLA), are caused by an expansion of a polyglutamine-coding CAG repeat within the respective genes. Various nonataxic signs are often presented, including ophthalmoplegia, saccadic abnormalities, pyramidal signs, extrapyramidal features, polyneuropathy, amyotrophy, fasciculation, dementia, and macular degeneration [1-3]. In 25 years, various SCA types have been identified and assigned as SCA1 to SCA40 by the Human Genome Nomenclature Committee [2, 4-7] with an estimated prevalence of each SCA being $<4$ per 100,000 population [8-12]. However, SCA3, more commonly known as Machado-Joseph disease (MJD), is found more prevalently worldwide [13-15]. Phenotypes of different SCAs often overlap and are indistinguishable, making a clinical diagnosis difficult. Several diagnostic genetic tests relating to clinical clues and epidemiological data of common SCAs have been developed and studied. In Thailand, MJD is the most common SCA type, followed by SCA1, SCA2, SCA6, and SCA17 [16, 17], by contrast with SCA7 and DRPLA, which are rare. Among Thai patients, MJD, SCA1, SCA2, and SCA6 mostly present a similar nonataxic phenotype with pyramidal features, predominantly in the lower limbs, and saccadic abnormalities, while peripheral neuropathy is less often presented [18].

The objectives of our study were to describe the epidemiological, clinical, and genotypic features of SCA in Buriram province in northeastern Thailand and to study 2 associations: first, between syndromic features and the genotype of SCA, and second, between health determinants and scores on the scale for the assessment and rating of ataxia (SARA) indicating severity.

\section{Materials and methods}

\section{Descriptive study}

After approval of our study protocol by the Ethical Committee on Human Rights Related to Research Involving Human Subjects, Buriram Hospital (ref. No. BR.0027.102.3/199) and obtaining informed consent from each patient participant, we conducted a cross-sectional observational study of 24 patients with autosomal dominant SCA from 13 families who were recruited from Buriram province in northeast Thailand between December 2009 and January 2014. Each patient gave a detailed clinical history and was examined by an experienced neurologist.
The profile and clinical data for each patient included sex, family hometown, age at onset, disease duration, age at diagnosis, family pedigree, history of smoking or alcohol drinking, eye movement abnormalities, nystagmus, cerebellar signs, fundoscopic examination, muscle tone and power, tendon reflexes, Babinski sign, parkinsonism, dystonia, chorea, dementia (screened by Mini-Mental State Examination), fasciculation, sensory examination testing for vibration, joint position and pinprick sensation, and SARA score.

\section{Laboratory study}

DNA samples were extracted from peripheral blood leukocytes using a phenol-chloroform method or a Qiagen DNA purification kit. Fluorescent-labeled polymerase chain reaction (PCR) amplification of the expanded repeat alleles of 6 genes, ataxin-1 (ATXN1), ataxin-2 (ATXN2), ataxin-3 (ATXN3), $\alpha-1 \mathrm{~A}$ subunit, $\mathrm{P} / \mathrm{Q}$ type voltage-dependent calcium channel (CACNA1A: SCA6), ataxin-7, and atrophin-1 (ATN1; DRPLA), was performed using primer sets from previously described methods [19-23]. The allele sizes were then determined by running the PCR products on a CEQ 8800 Genetic Analysis System (Beckman Coulter). Sizes of CAG repeats of both normal and pathological alleles of each chromosome were calculated by comparing results with the size of a normal control sample, of which the sizes were previously defined by direct Sanger sequencing. To avoid a false negative result caused by a very large expanded allele failing to be amplified by using regular PCR [24], all samples whose results showed patterns of homozygous wild-type alleles of $A T X N 1, A T X N 2$, and $A T X N 3$ were subsequently reanalyzed by performing long-range PCR using TaKaRa LA Taq polymerase.

\section{Analytical study and statistical analyses}

All statistical analyses were conducted using SPSS for Windows version 11.0. The mean, proportion, and range were determined. Chi-square and unpaired $t$ tests were used to analyze possible associations between the type of SCA and sex, age, family history, and clinical features. Furthermore, we analyzed associations between the type of SCA and any underlying disease, age at onset, body weight, smoking status, family history, alcohol consumption, sex, head injury history, and SARA. $P<0.05$ was considered significant. 


\section{Results}

\section{Epidemiological findings}

Buriram province in northeast Thailand is divided geographically into 22 districts (amphoes). All families with SCA living in Buriram province have either SCA1 or MJD (SCA3) (Figure 1). In 13 autosomal dominant families with SCA, 7 (54\%) tested positive for SCA1, 6 (46\%) for MJD, and none for SCA2 and SCA6. This suggests that MJD and SCA1 are more common in the autosomal dominant families with ataxia in Buriram. A total of 24 index patients from Table 1 ( 3 of 13 SCA1 cases did not have a family history) were enrolled in the study, including 11 patients with MJD (46\%) and 13 with SCA1 (54\%).

The average age of the recruited patients was 43.7 years (range 20-72 years), whereas the average age at disease onset was 36.9 years (range $20-59$ years). Overall patient characteristics, including sex, family history, clinical features including slow saccade, upward gaze paresis, horizontal nystagmus, vertical nystagmus, pale optic disc, hyperreflexia, Babinski sign, areflexia, sensory impairment, and parkinsonism, are summarized in Table 1.
Table 1. Characteristics of spinocerebellar ataxia types in Buriram province, northeast Thailand

\begin{tabular}{lccc}
\hline Characteristic, $\mathbf{n}(\%)$ & SCA1 & MJD (SCA3) & $\boldsymbol{P}$ \\
\hline Cases (n) & 13 & 11 & \\
Sex & & & \\
Male & $5(39)$ & $7(64)$ & 0.41 \\
Female & $8(61)$ & $4(36)$ & \\
Mean age of onset, years (range) & $37.0(18-56)$ & $38.6(25-59)$ & 0.28 \\
Positive family history & $10(77)$ & $11(100)$ & 0.22 \\
Clinical features & & & \\
Slow saccade & $9(69)$ & $9(82)$ & 0.65 \\
Upward gaze paresis* & $3(23)$ & $9(82)$ & 0.012 \\
Horizontal nystagmus* & $3(23)$ & $10(91)$ & 0.001 \\
Vertical nystagmus & $1(8)$ & $3(27)$ & 0.30 \\
Pale optic disc & 0 & 0 & \\
Hyperreflexia & $11(85)$ & $8(73)$ & 0.63 \\
Babinski sign & $1(8)$ & $2(18)$ & 0.58 \\
Areflexia & $1(8)$ & $3(27)$ & 0.30 \\
Sensory impairment & $2(15)$ & $5(46)$ & 0.18 \\
Parkinsonism & $2(15)$ & $1(9)$ & $>0.99$ \\
\hline
\end{tabular}

${ }^{*} P<0.05$

MJD, Machado-Joseph disease; SCA, spinocerebellar ataxia; SCA1, spinocerebellar ataxia type 1; SCA3, spinocerebellar ataxia type 3

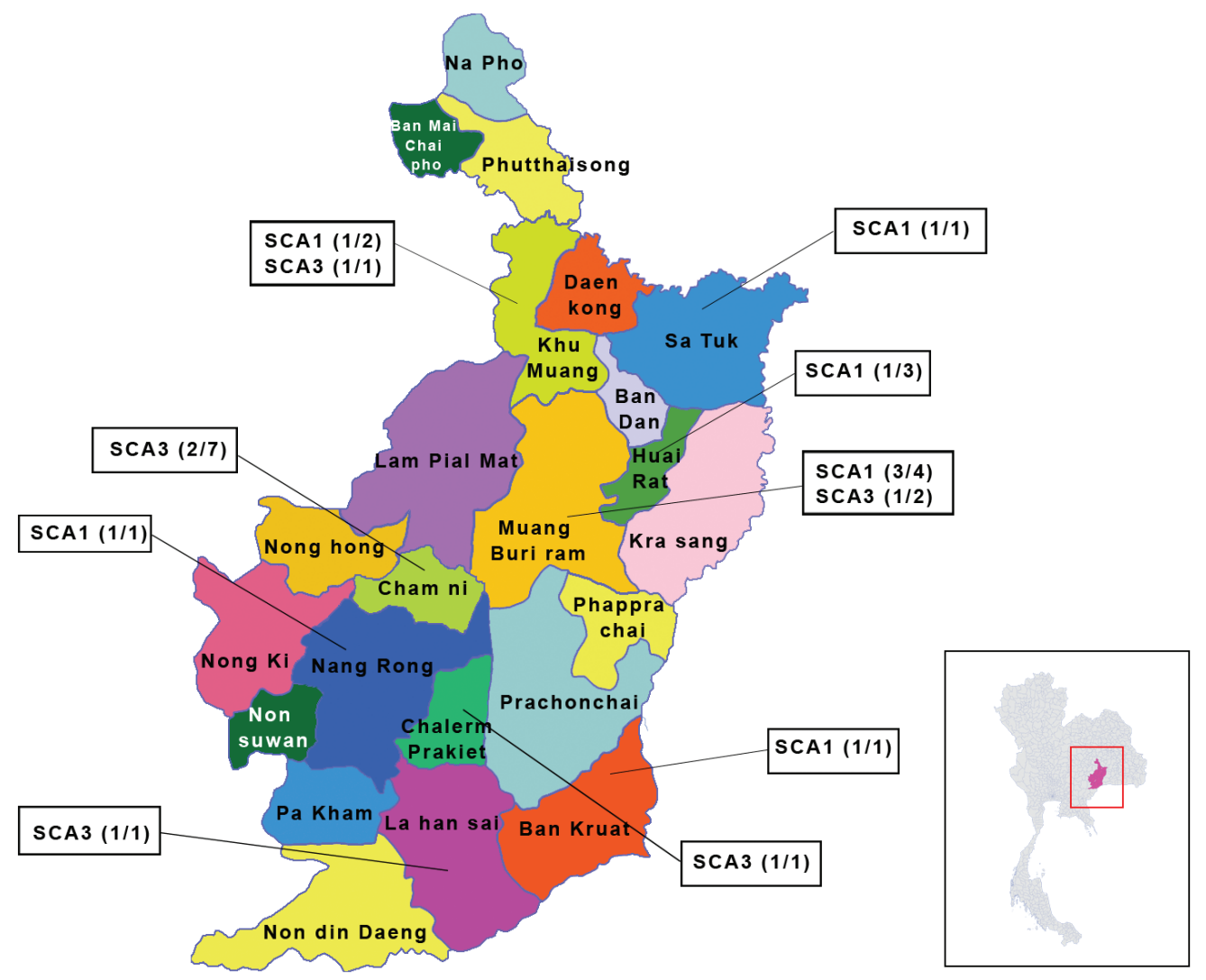

Figure 1. Distribution of spinocerebellar ataxias in Buriram province, northeast Thailand (families/number of cases). MJD, Machado-Joseph disease; SCA1, spinocerebellar ataxia type 1; SCA3, spinocerebellar ataxia type 3 (MJD) 


\section{Clinical features in patients with SCA}

All patients with SCA showed gait ataxia as a presenting symptom of the disease; gait problems were earlier in onset and more severely affected than speech problems. Other cerebellar features, such as arm incoordination and cerebellar dysarthria, commonly followed within a few years. Mildly asymmetrical limb ataxia was present in most cases. In advanced stages of the disease, truncal ataxia was present in all cases leading to bed-bound disability.

In the early stages, pyramidal signs, predominantly increased tendon reflexes and spastic tone in both legs, could frequently be detected as hyperreflexia was evident in most cases $(79 \%)$. Areflexia was seen in 4 patients $(16 \%)$. Babinski sign was found in only $3(13 \%)$ patients. The presence of any pyramidal signs was not significantly different between MJD and SCA1. Extrapyramidal features appeared to be uncommon in the patients studied. Parkinsonism was observed in a few cases, while chorea and dystonia was not detected in any of the cases (if parkinsonism is found only at a late stage of the disease or in patients with long disease duration, already severely ataxic with mild parkinsonism, it may be worth to mention, because at the later stage, more system involvement would be observed; for clinical clues at an early stage, this sign may not be helpful).

\section{Ophthalmoparesis features}

Gaze-evoked nystagmus was a common sign. Horizontal nystagmus was more common than vertical nystagmus. Horizontal nystagmus and upward gaze paresis were significantly associated with MJD (Table 1). Abnormal saccadic eye movements were demonstrated in both subtypes of SCAs. Slow saccades were found in about $18(75 \%)$ of the patients. Slow saccades were not different between SCA1 and MJD $(P=0.65)$. Impairment of the accuracy of the saccade or dysmetric saccade was also identified in most patients for both SCA subtypes.

In comparing the clinical features of study participants, 15 SCA patients had SARA $\geq 15 ; 7$ of these cases were SCA1 and 8 were MJD. Demographic data such as sex, family history of SCA, underlying medical problems, onset age $>35$ years, body weight $>60 \mathrm{~kg}$, smoking, and alcohol drinking were compared between the 2 groups based on SARA score. There were no significant differences in demographic data between the groups with SARA $\geq 15$ and SARA $<15$ (Table 2).
Table 2. Comparison of demographic data and clinical factors between patients with SARA $\geq 15$ and SARA $<15$

\begin{tabular}{lccc}
\hline Data or factor, $\mathbf{n}(\%)$ & SARA $\geq \mathbf{1 5}(\mathbf{n}=\mathbf{1 5})$ & SARA $<\mathbf{1 5}(\mathbf{n}=\mathbf{9})$ & $\boldsymbol{P}$ \\
\hline SCA1 & $7(47)$ & $6(67)$ & \\
MJD (SCA3) & $8(53)$ & $3(33)$ & 0.34 \\
Male & $7(47)$ & $5(56)$ & 0.67 \\
Female & $8(53)$ & $4(44)$ & \\
Family history of SCA & $14(93)$ & $7(78)$ & \\
No family history & $1(7)$ & $2(22)$ & 0.27 \\
Medical diseases ${ }^{\dagger}$ & $7(47)$ & $3(33)$ & \\
No medical disease & $8(53)$ & $6(67)$ & 0.52 \\
Onset age $\geq 35$ years & $9(60)$ & $5(56)$ & \\
Onset age $<35$ years & $6(40)$ & $4(44)$ & 0.83 \\
Body weight $\geq 60$ kg & $8(53)$ & $3(33)$ & \\
Body weight $<60$ kg & $7(47)$ & $6(67)$ & 0.34 \\
Smoking & $5(33)$ & $4(44)$ & \\
Nonsmoking & $10(67)$ & $5(56)$ & 0.59 \\
Alcohol drinking & $7(47)$ & $7(78)$ & \\
Nonalcohol drinking & $8(53)$ & $2(22)$ & 0.14 \\
Head injury & $1(7)$ & $1(11)$ & 0.70 \\
No history of head injury & $14(93)$ & $8(89)$ & \\
\hline
\end{tabular}

${ }^{+}$Medical diseases, diabetes mellitus and hypertension MJD, Machado-Joseph disease; SARA, scale for the assessment and rating of ataxia; SCA, spinocerebellar ataxia; SCA1, spinocerebellar ataxia type 1; SCA3, spinocerebellar ataxia type 3

\section{Discussion}

MJD (SCA3) was found in 11 patients from 6 of the 13 families with SCA, giving a frequency of $46 \%$ among SCA patients in the present study with limited patient recruitment. The apparent lower prevalence of MJD compared with SCA1 in Buriram is different from that in the general Thai population, in which MJD is the most common SCA, similar to the prevalence in other East Asian countries, except Korea in which SCA2 appears to be the most common SCA [18, 25]. Moreover, no patients had SCA2 or SCA6. Further study of individuals with SCA in Buriram province, northeast Thailand, might find a different proportion of MJD and SCA1 than in other regions of Thailand and other countries.

The clinical phenotypes of SCA1, SCA2, SCA3 (MJD), and SCA6 have been firmly established. Some evidence suggested that the larger size of the CAG repeats is more likely to be associated with early age at onset [26]. In all SCAs, progressive ataxia is the predominant clinical manifestation. However, patients with SCA1, SCA2, and MJD frequently present with additional nonataxic symptoms [27]. Although the associated nonataxic symptoms, such as slow saccade and 
pyramidal signs, were associated in some degree with certain types of these SCAs, the associations often varied among different studies, suggesting that other genetic factors or staging of the diseases might influence the phenotypic expression. Pathological nystagmus is frequently oculomotor alteration of patients with MJD in a region of Brazil [28]. In this study, ophthalmoparesis, such as upward gaze paresis and horizontal nystagmus, was significantly more common in MJD than in other SCAs [18]. The clinical features of eye movement abnormalities that are different between MJD and SCA1 among studied families may be caused by factors of sharing some common ancestor and having similar other influential genetic background compared with those in the previous large study in Thailand, in which only patients with index cases from different families were recruited [18]. No significant differences in the health determinants were found between the groups with $\mathrm{SARA} \geq 15$ and $\mathrm{SARA}<15$.

The present study has some limitations, especially for neurological examinations and blood tests for all studied family members in their communities. The sample size was relatively small and perhaps not sufficiently large enough to explain the differences of predisposing factors and related medical history among patients. A large proportion of our patients were of low socioeconomic and low education status. This might result in inaccurate data for some important information. Family planning and routine medical checks are recommended for these patients and their families.

\section{Conclusion}

MJD (SCA3) and SCA1 were the 2 adult-onset cerebellar degenerative diseases found in Buriram province with identified geographical distribution. The patients with MJD or SCA1 often exhibited a similar nonataxic phenotype, including pyramidal features (hyperreflexia), predominantly in the lower limbs; slow saccadic abnormalities; and, less often, peripheral neuropathy. Ophthalmoparesis, including upward gaze paresis and horizontal nystagmus, was significantly more common in MJD than it was in SCA1. These 2 clinical signs should be considered as a helpful clue for differential diagnosis of SCA subtypes, at least in the Buriram province in Thailand.

Author contributions. SW and TP contributed substantially to the conception and design of the study. AM substantially collected the data, and $\mathrm{CP}$ contributed to their interpretation. All authors were involved in drafting the manuscript and its critical revision, approved the final version submitted, and take responsibility for the statements made in the published article.

Acknowledgments. We are grateful to all participants and their family members. We acknowledge Dr. Soraya Kaewpitoon (Suranaree University of Technology) and Dr. Patcharee Yimrattanabowon and Miss Chutima Deesawat at Buriram Hospital for their help. This study was supported by the Neurogenetic Fund (3001180) of the Ramathibodi Foundation.

Conflicts of interest. The authors have each completed and submitted an International Committee of Medical Journal Editors Uniform Disclosure Form for Potential Conflicts of Interest. None of the authors disclose any conflict of interest.

\section{References}

[1] Durr A. Autosomal dominant cerebellar ataxias: polyglutamine expansions and beyond. Lancet Neurol. 2010; 9:885-94.

[2] Klockgether T, Paulson H. Milestones in ataxia. Mov Disord. 2011; 26:1134-41.

[3] Paulson HL. The spinocerebellar ataxias. J Neuroophthalmol. 2009; 29:227-37.

[4] Tsoi H, Yu AC, Chen ZS, Ng NK, Chan AY, Yuen LY, et al. A novel missense mutation in CCDC88C activates the JNK pathway and causes a dominant form of spinocerebellar ataxia. J Med Genet. 2014; 51:590-5.

[5] Kobayashi H, Abe K, Matsuura T, Ikeda Y, Hitomi T, Akechi Y, et al. Expansion of intronic GGCCTG hexanucleotide repeat in NOP56 causes SCA36, a type of spinocerebellar ataxia accompanied by motor neuron involvement. Am J Hum Genet. 2011; 89:121-30.

[6] Serrano-Munuera C, Corral-Juan M, Stevanin G, San Nicolás H, Roig C, Corral J, et al. New subtype of spinocerebellar ataxia with altered vertical eye movements mapping to chromosome $1 \mathrm{p} 32$. JAMA Neurol. 2013; 70:764-71.

[7] Wang JL, Yang X, Xia K, Hu ZM, Weng L, Jin X, et al. TGM6 identified as a novel causative gene of spinocerebellar ataxias using exome sequencing. Brain. 2010; 133:3510-8.

[8] Ashizawa T, Figueroa KP, Perlman SL, Gomez CM, Wilmot GR, Schmahmann JD, et al. Clinical characteristics of patients with spinocerebellar ataxias 1, 2, 3 and 6 in the US; a prospective observational study. Orphanet J Rare Dis. 2013; 8:177. doi: 10.1186/1750-1172-8-177

[9] Paulson H. Machado-Joseph disease/spinocerebellar ataxia type 3. In: Subramony SH, Dürr A, editors. Handbook of clinical neurology. Vol. 103 (3rd series). Ataxic disorders. Amsterdam: Elsevier; 2012, p. 437-49.

[10] Pulst SM. Spinocerebellar ataxia type 2. In: Pagon RA, Adam MP, Ardinger HH, Wallace SE, Amemiya A, Bean LJH, et al., editors. GeneReviews [Internet]. Seattle: University of Washington; 1998 [updated 2015 Nov 12; cited 2017 Feb 28]. Available at: https://www. ncbi.nlm.nih.gov/books/NBK1275/. 
[11] Solodkin A, Gomez CM. Spinocerebellar ataxia type 6. In: Subramony SH, Dürr A, editors. Handbook of clinical neurology. Vol. 103 (3rd series). Ataxic disorders. Amsterdam: Elsevier; 2012, p. 461-73.

[12] van de Warrenburg BP, Sinke RJ, Verschuuren-Bemelmans CC, Scheffer H, Brunt ER, Ippel PF, et al. Spinocerebellar ataxias in the Netherlands: prevalence and age at onset variance analysis. Neurol. 2002; 58:702-8.

[13] Martins S, Calafell F, Gaspar C, Wong VC, Silveira I, Nicholson GA, et al. Asian origin for the worldwide-spread mutational event in Machado-Joseph disease. Arch Neurol. 2007; 64:1502-8.

[14] Takano H, Cancel G, Ikeuchi T, Lorenzetti D, Mawad R, Stevanin $\mathrm{G}$, et al. Close associations between prevalences of dominantly inherited spinocerebellar ataxias with CAG-repeat expansions and frequencies of large normal CAG alleles in Japanese and Caucasian populations. Am J Hum Genet. 1998; 63:1060-6.

[15] Tang B, Liu C, Shen L, Dai H, Pan Q, Jing L, et al. Frequency of SCA1, SCA2, SCA3/MJD, SCA6, SCA7, and DRPLA CAG trinucleotide repeat expansion in patients with hereditary spinocerebellar ataxia from Chinese kindreds. Arch Neurol. 2000; 57:540-4.

[16] Choubtum L, Witoonpanich P, Hanchaiphiboolkul S, Bhidayasiri R, Jitkritsadakul O, Pongpakdee S, et al. Analysis of SCA8, SCA10, SCA12, SCA17 and SCA19 in patients with unknown spinocerebellar ataxia: a Thai multicentre study. BMC Neurol. 2015; 15:166. doi: 10.1186/s12883-015-0425-y

[17] Sura T, Eu-Ahsunthornwattana J, Youngcharoen S, Busabaratana M, Dejsuphong D, Trachoo O, et al. Frequencies of spinocerebellar ataxia subtypes in Thailand: window to the population history? J Hum Genet. 2009; 54:284-8.

[18] Boonkongchuen P, Pongpakdee S, Jindahra P, Papsing C, Peerapatmongkol P, Wetchaphanphesat $\mathrm{S}$, et al. Clinical analysis of adult-onset spinocerebellar ataxias in Thailand. BMC Neurol. 2014; 14:75. doi: 10.1186/1471-2377-14-75

[19] Giunti P, Sabbadini G, Sweeney MG, Davis MB, Veneziano L, Mantuano E, et al. The role of the SCA2 trinucleotide repeat expansion in 89 autosomal dominant cerebellar ataxia families. Frequency, clinical and genetic correlates. Brain. 1998; 121:459-67.
[20] Giunti P, Sweeney MG, Harding AE. Detection of the MachadoJoseph disease/spinocerebellar ataxia three trinucleotide repeat expansion in families with autosomal dominant motor disorders, including the Drew family of Walworth. Brain. 1995; 118:1077-85.

[21] Giunti P, Sweeney MG, Spadaro M, Jodice C, Novelletto A, Malaspina $\mathrm{P}$, et al. The trinucleotide repeat expansion on chromosome 6p (SCA1) in autosomal dominant cerebellar ataxias. Brain. 1994; 117:645-9.

[22] Warner TT, Williams LD, Walker RW, Flinter F, Robb SA, Bundey SE, et al. A clinical and molecular genetic study of dentatorubropallidoluysian atrophy in four European families. Ann Neurol. 1995; 37:452-9.

[23] Zhuchenko O, Bailey J, Bonnen P, Ashizawa T, Stockton DW, Amos $\mathrm{C}$, et al. Autosomal dominant cerebellar ataxia (SCA6) associated with small polyglutamine expansions in the $\alpha_{1 \mathrm{~A}}$-voltage-dependent calcium channel. Nat Genet. 1997; 15:62-9.

[24] Gan SR, Shi SS, Wu JJ, Wang N, Zhao GX, Weng ST, et al. High frequency of Machado-Joseph disease identified in Southeastern Chinese kindreds with spinocerebellar ataxia. BMC Med Genet. 2010; 11:47. doi: 10.1186/1471-2350-11-47

[25] Maruyama H, Izumi Y, Morino H, Oda M, Toji H, Nakamura $\mathrm{S}$, et al. Difference in disease-free survival curve and regional distribution according to subtype of spinocerebellar ataxia: a study of 1,286 Japanese patients. Am J Med Genet. 2002; 114:578-83.

[26] Laffita-Mesa JM, Bauer PO, Kouri V, Pena Serrano L, Roskams J, Almaguer Gotay D, et al. Epigenetics DNA methylation in the core ataxin-2 gene promoter: novel physiological and pathological implications. Hum Genet. 2012; 131:625-38.

[27] Dürr A, Stevanin G, Cancel G, Duyckaerts C, Abbas N, Didierjean $\mathrm{O}$, et al. Spinocerebellar ataxia 3 and Machado-Joseph disease: clinical, molecular, and neuropathological features. Ann Neurol. 1996; 39:490-9.

[28] Jardim LB, Pereira ML, Silveira I, Ferro A, Sequeiros J, Giugliani R. Neurologic findings in Machado-Joseph disease: relation with disease duration, subtypes, and (CAG) $)_{n}$ Arch Neurol. 2001; 58:899-904. 